NOTE

\title{
Measurement of skin dose variations produced by a silicon-based protective dressing in radiotherapy
}

\author{
Martin J Butson ${ }^{1,2}$, Tsang Cheung ${ }^{1}$, Peter K N Yu${ }^{1}$ and Peter Metcalfe ${ }^{2}$ \\ ${ }^{1}$ Department of Physics and Materials Science, City University of Hong Kong, Kowloon Tong, \\ Hong Kong, People's Republic of China \\ 2 Department of Medical Physics, Illawarra Cancer Care Centre, Crown St, Wollongong, \\ NSW 2500, Australia \\ E-mail: mbutson@guessmail.com
}

Received 13 February 2002

Published 22 May 2002

Online at stacks.iop.org/PMB/47/N145

\begin{abstract}
Variations in skin dose caused by a silicon-based burn dressing used in radiotherapy during treatment have been investigated. Measurement of these variations in skin dose has been achieved using thermoluminescent dosimeters (TLDs) and Gafchromic film. For a $6 \mathrm{MV}$ x-ray beam results have shown that an approximately $0.4 \mathrm{~mm}$ thick silicon mesh dressing increases the average surface dose by approximately $12.5 \%$ to $14 \%$ of the maximum and average dose at $1 \mathrm{~mm}$ depth and by $4 \%$ to $6 \%$ of the maximum for field sizes ranging from $5 \mathrm{~cm} \times 5 \mathrm{~cm}$ up to $40 \mathrm{~cm} \times 40 \mathrm{~cm}$ at $100 \mathrm{~cm}$ source to surface distance (SSD). The radiation effective thickness of the silicon dressing was calculated to be $0.5 \mathrm{~mm} \pm 0.05 \mathrm{~mm}$ water equivalent. TLDs of various thicknesses provide point-dose assessment and Gafchromic film can provide a detailed twodimensional dose map with a high spatial resolution. Results have shown that a large variation in skin dose is delivered under the dressing depending on the amount of material directly above it as defined by the silicon mesh outline.
\end{abstract}

\section{Introduction}

Skin dose can vary quite considerably within the first few millimetres of depth due to the buildup characteristics of x-ray beams. These changes can be attributed to variations in electron contamination caused by parameters such as the field size, the use of beam-modifying devices and factors involved with in-phantom scatter such as exit dose and beam angle of incidence (Butson et al 1996, Zhu and Palta 1998, Hounsell and Wilkinson 1999). A silicon-based burn dressing can be used as a protective layer for skin during radiotherapy. This dressing may reduce skin damage caused by excess friction or rubbing of the irradiated area on clothes or other materials during treatment (Thilmann et al 1996a, 1996b). The use of such a dressing, which is normally left in place for an extended period of time, introduces scatter material in 
the beam's path during irradiation, similar to a bolus. This note examines the variations in skin dose caused by this material for $6 \mathrm{MV}$ x-rays as an integrated dose average increase and over a two-dimensional (2D) area.

\section{Materials and methods}

Measurements were made using a Varian 2100C linear accelerator, $6 \mathrm{MV}$ x-rays. A fixed SSD of $100 \mathrm{~cm}$ was used for analysis of skin dose variations. Point-dose measurements were performed using lithium fluoride thermoluminescent dosimeters (TLDs) and an Attix parallel plate ionization chamber. Profile and 2D map measurements were made using MD-55 Gafchromic film. This was performed by placing the detectors in a solid water slab phantom $(30 \mathrm{~cm} \times 30 \mathrm{~cm} \times 30 \mathrm{~cm})$ during irradiation. The phantom was constructed from various slices of solid water that ranged in thickness from $1 \mathrm{~mm}$ to $2 \mathrm{~cm}$ which allowed the detectors to be placed at various depths. Experiments for measurement of dose at $6 \mathrm{MV}$ x-rays are performed with field sizes ranging from $5 \mathrm{~cm} \times 5 \mathrm{~cm}$ to $40 \mathrm{~cm} \times 40 \mathrm{~cm}$, with and without the Mepitel dressing placed over the phantom as would be the case clinically. Results at central axis for various depths from the surface to $1 \mathrm{~mm}$ depth are measured as well as profile and 2D maps using the Gafchromic film. Results are quoted for the percentage dose of maximum. This is performed by comparing the doses measured by the detectors at the depth of interest to the dose at the depth of maximum dose. The authors acknowledge that percentage doses in the build-up region and especially at the surface vary considerably with variations in the clinical set-up such as field size, SSD and phantom shape. The authors have quoted data this way as a representative guide to the effects seen by the introduction of the silicon dressing.

The TLDs used were Harshaw lithium fluoride (LiF) thermoluminescent dosimeters. The TLDs were held in tissue equivalent packaging, which was placed at the surface of a solid water (Constantinou et al 1982) phantom for skin dose analysis. The TLDs used had three different thicknesses of $0.038,0.099$ and $0.23 \mathrm{~g} \mathrm{~cm}^{-2}$ (as stated by the manufacturer). All the chips have the same surface area of $3.15 \mathrm{~mm} \times 3.15 \mathrm{~mm}$. By comparing their physical densities and by assuming that the effective point of measurement is in the centre of each chip nominal thicknesses or effective depths of measurement for the three chips are $0.14 \mathrm{~mm}$, $0.39 \mathrm{~mm}$ and $0.89 \mathrm{~mm}$ (Kron et al 1993). Results obtained with these chips provide skin dose information within the first $1 \mathrm{~mm}$. The detectors were read out in a NE Rialto reader using a $160{ }^{\circ} \mathrm{C}$ for $10 \mathrm{~s}$ pre-read followed by a $300{ }^{\circ} \mathrm{C}$ read cycle for $15 \mathrm{~s}$. All the chips were annealed $\left(1 \mathrm{~h} 400{ }^{\circ} \mathrm{C}\right.$ followed by $\left.2 \mathrm{~h} 100{ }^{\circ} \mathrm{C}\right)$ in a dedicated TLD annealing oven (PTW, Freiburg, FRG, type TLD0) after each readout). The precision of standard dose measurements using these chips (two standard deviations) was 6\%, 3\% and 3\% for the chips with nominal thicknesses of $0.14 \mathrm{~mm}, 0.39 \mathrm{~mm}, 0.89 \mathrm{~mm}$, respectively, at $100 \mathrm{cGy}$ applied dose. The Attix parallel plate ionization chamber is constructed primarily from solid water to closely approximate the interaction properties of water itself. The chamber window consists of a $0.025 \mathrm{~mm}$ thick, $4.8 \mathrm{mg} \mathrm{cm}^{-1}$ dense Kapton conductive film. The conducting surfaces are made up of minimal thickness colloidal graphite. The air gap is $1 \mathrm{~mm}$ giving an ionization collecting volume of approximately $0.127 \mathrm{~cm}^{3}$, and a sensitive collection area of $1.27 \mathrm{~cm}^{2}$, which is vented to the atmosphere. Its over response due to side wall scatter is less than 1\% (Rawlinson 1992). The MD-55-2 Gafchromic film (ISP Technologies Inc.) used was from batch number 970116. Precautions outlined in TG-55 for handling the radiochromic film were followed (Niroomand Rad et al 1998). The film during its storage and analysis was maintained in the temperature range of $22{ }^{\circ} \mathrm{C} \pm 2{ }^{\circ} \mathrm{C}$ thus reducing the effects of time and temperature-dependent evolution and readout (Meigooni et al 1996) of the absorption spectra of the film. The film is only removed from a light tight envelope during irradiation and readout 


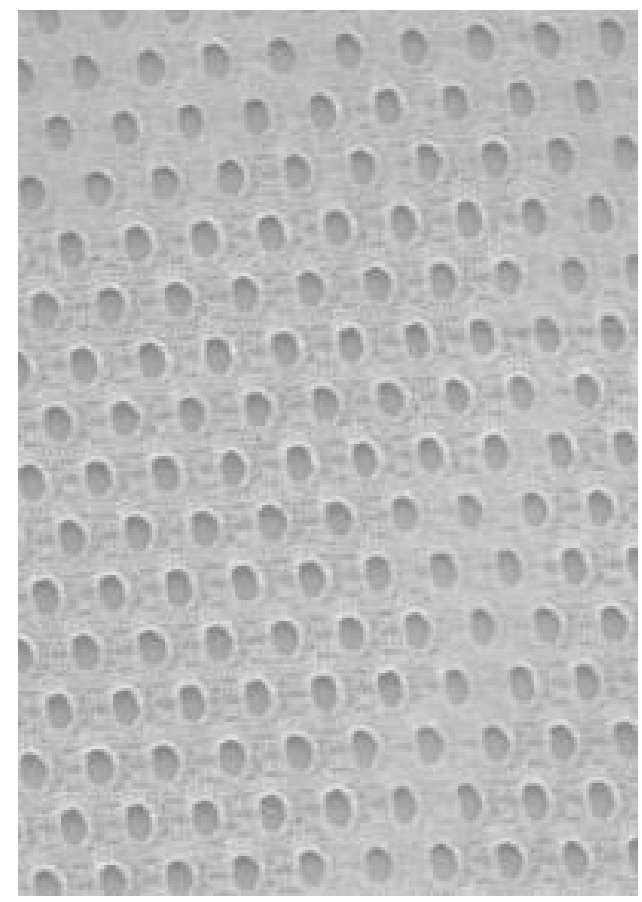

Figure 1. Silicon-based Mepitel burn dressing.

to reduce the effects of ambient light (Butson et al 1998). The film was calibrated using a double exposure technique where the optical density of the film detector is first recorded for no absorbed dose. Then a standard dose of $5 \mathrm{~Gy}$ was delivered and optical density over the entire film assessed for dose response. The film is initially assessed for background optical density (OD) which is then subtracted from results producing a net OD for irradiated doses. Variations in the background OD were found to be $\pm 3 \%$ over the film pieces. Corrections were applied accordingly. Optical density was assessed using a $660 \mathrm{~nm}$ readout wavelength densitometer (Carolan et al 1997).

The silicon dressing material used was Mepitel (Williams 1995, Vloemens et al 1994) silicon dressing (SCA Molnlycke Ltd). Mepitel consists of an elastic transparent polyamide net, which is impregnated with a medical grade cross-linked silicone that encloses each fibre of the polyamide net. The dressing, which is non-absorbent and inert, is apertured to allow the passage of wound exudate and thereby also allowing the skin to breathe. A close-up picture of the Mepitel dressing is shown in figure 1. The spacing between the open circular holes in the dressing mesh is approximately $3 \mathrm{~mm}$ and the dressing is approximately $0.4 \mathrm{~mm}$ thick. Approximate dimensions are given as the dressing is very flexible and malleable for use on irregular surfaces and can be stretched which distorts the shape of the mesh pattern.

\section{Results and discussion}

Table 1 shows the percentage dose build-up curves for an open field and a field with the Mepitel dressing applied for a $20 \mathrm{~cm} \times 20 \mathrm{~cm}$ field size. Also shown is the percentage dose difference between the two curves at the specified depths. Results show that the major differences in delivered dose occur within the first $3-4 \mathrm{~mm}$ depth. This is due to the large dose gradients 


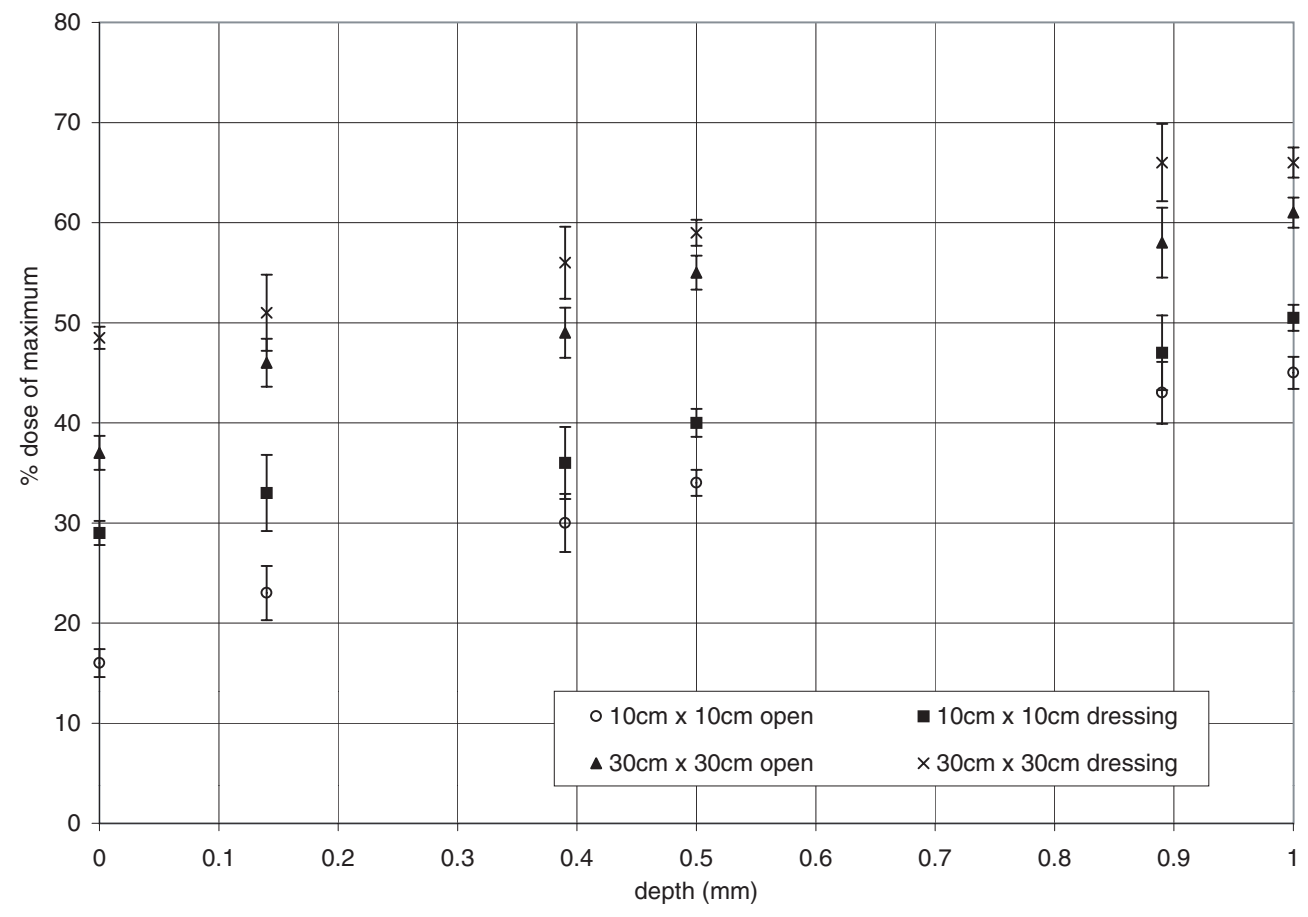

Figure 2. Build-up curves within the first $1 \mathrm{~mm}$ depth for $\mathrm{x}$-ray field, with and without the Mepitel dressing.

Table 1. Percentage dose build-up curves for a $20 \mathrm{~cm} \times 20 \mathrm{~cm}$ field with and without the Mepitel dressing.

\begin{tabular}{llll}
\hline Depth $(\mathrm{mm})$ & $\begin{array}{l}\text { Open field } \\
\left(\% \text { of } D_{\max }\right)\end{array}$ & $\begin{array}{l}\text { Mepitel dressing } \\
\left(\% \text { of } D_{\max }\right)\end{array}$ & $\begin{array}{l}\text { Difference } \\
\left(\% \text { of } D_{\max }\right)\end{array}$ \\
\hline 0 & 27 & 39 & 12 \\
1 & 53 & 59 & 6 \\
2 & 69 & 73 & 4 \\
3 & 79 & 82 & 3 \\
4 & 85 & 87 & 2 \\
5 & 91 & 92 & 1 \\
7 & 95 & 96 & 1 \\
10 & 98.5 & 99 & 0.5 \\
12 & 99.5 & 99.5 & 0 \\
14 & 100 & 100 & 0 \\
\hline
\end{tabular}

occurring in this region. The extra scatter material created by the Mepitel dressing in effect produces a thin bolus. Figure 2 shows results for open build-up curves within the first $1 \mathrm{~mm}$ for open fields and with the silicon dressing at $10 \mathrm{~cm} \times 10 \mathrm{~cm}$ and $30 \mathrm{~cm} \times 30 \mathrm{~cm}$ field sizes. The results shown are a combination of TLD and Attix chamber results. Points at $0 \mathrm{~mm}, 0.5 \mathrm{~mm}$ and $1 \mathrm{~mm}$ are the results for Attix chamber and at $0.14 \mathrm{~mm}, 0.39 \mathrm{~mm}$ and $0.89 \mathrm{~mm}$ are TLD results. These results show that the dressing has produced an overall increase in surface dose by $13 \%$ and $11.5 \%$ of the maximum at $10 \mathrm{~cm} \times 10 \mathrm{~cm}$ and $30 \mathrm{~cm} \times$ $30 \mathrm{~cm}$ field sizes, respectively, with this change decreasing to $5.5 \%$ and $5 \%$ at $1 \mathrm{~mm}$ depth. The larger errors seen for the TLD results are assumed to be due to a combination of two 
Table 2. Measured dose at surface and $1 \mathrm{~mm}$ depth for open fields and with the silicon dressing.

\begin{tabular}{|c|c|c|c|c|}
\hline \multirow[b]{2}{*}{$\begin{array}{l}\text { Field size } \\
(\mathrm{cm} \times \mathrm{cm})\end{array}$} & Open & Dressing & Open & Dressing \\
\hline & \multicolumn{2}{|c|}{$\begin{array}{l}\text { Surface dose } \\
\text { (\% of max) }\end{array}$} & \multicolumn{2}{|c|}{$\begin{array}{l}1 \mathrm{~mm} \text { depth dose } \\
(\% \text { of } \max )\end{array}$} \\
\hline 5 & 10 & 24 & 40 & 46 \\
\hline 10 & 16 & 29 & 45 & 50.5 \\
\hline 20 & 27 & 39 & 53 & 59 \\
\hline 30 & 37 & 48.5 & 61 & 66 \\
\hline 40 & 42 & 54.5 & 66 & 70 \\
\hline
\end{tabular}

effects. Firstly, due to the lower accuracy of TLD standard dose assessment and secondly due to variations in build-up dose seen over a small area when using the Mepitel dressing. The TLDs have a surface dimension of approximately $3 \mathrm{~mm} \times 3 \mathrm{~mm}$. The dimension of the silicon dressing between the centre of each hole is approximately $2.5 \mathrm{~mm}$ and the holes are approximately $1.2 \mathrm{~mm}$ in diameter. This means that approximately $20 \%$ of the dressing is open and $80 \%$ is solid material. The position of the TLD chip during irradiation with respect to the silicon dressing (i.e. mainly over a hole or mainly over a solid area) can affect the measured dose within the first $1 \mathrm{~mm}$. It is very difficult to align the TLDs with respect to the dressing, i.e. under the hole, on the dressing or under the solid material. Thus the increase in measurement error/variation will occur.

Table 2 shows the percentage of maximum dose with field size variations for open fields and with the dressing (at the surface and at $1 \mathrm{~mm}$ depth) as measured by the Attix parallel plate ionization chamber. The sensitive area of measurement for the Attix chamber is $1.27 \mathrm{~cm}^{2}$. This represents an area covering approximately 30 holes within the dressing and thus gives a relatively accurate average dose when using the Mepitel dressing. The silicon dressing provides a relatively uniform average increase in percentage dose of maximum for all field sizes and at all depths within the first $1 \mathrm{~mm}$. This is mainly due to the extra photon scatter produced from the silicon dressing itself, which acts similar to a thin bolus material. By comparing the build-up curves for open fields to fields with the dressing, an effective thickness of the dressing can be estimated. Results over the range of field sizes from $5 \mathrm{~cm} \times 5 \mathrm{~cm}$ to $40 \mathrm{~cm} \times 40 \mathrm{~cm}$ give the effective thickness of the dressing to be $0.5 \mathrm{~mm}$.

Figure 3 shows a dose profile measured near the surface with radiochromic film (effective depth of measurement is $0.17 \mathrm{~mm}$ ) through the silicon mesh material for a $10 \mathrm{~cm} \times 10 \mathrm{~cm}$ field-size. As can be seen a large variation occurs due to the presence of the mesh air gaps and thus a high degree of variation in the build-up material on a small spatial resolution. On this section of mesh, the percentage of $D_{\max }$ dose varies from approximately 25 to $32.5 \%$. The average dose is calculated as $29 \% \pm 4.5 \%$ ( 2 standard deviations of the mean) for this area. It produces a wave pattern with approximately $3 \mathrm{~mm}$ distance between the peaks that relate to the position of the solid 'silicon' in the mesh whereas the troughs relate to the 'holes' in the mesh. This pattern is more evident when shown over a 2D area as in figure 4 (for the same field). This highlights the peaks and troughs of the dose and forms a similar mesh structure in the dose when observed in this manner. The average dose calculated here is $29 \% \pm 5.5 \%$ (2 SD). The lower doses represent the areas covered by the holes in the dressing and the higher doses are the areas covered by the solid dressing material.

Although our centre has only begun trials for clinical use of the silicon dressing there seems to be a general feeling that the use of the dressing in areas which may be susceptible to radiation may benefit induced skin reactions such as erythema. Results have shown that an average increase in surface dose due to the Mepitel dressing is approximately $10-13.5 \%$ 


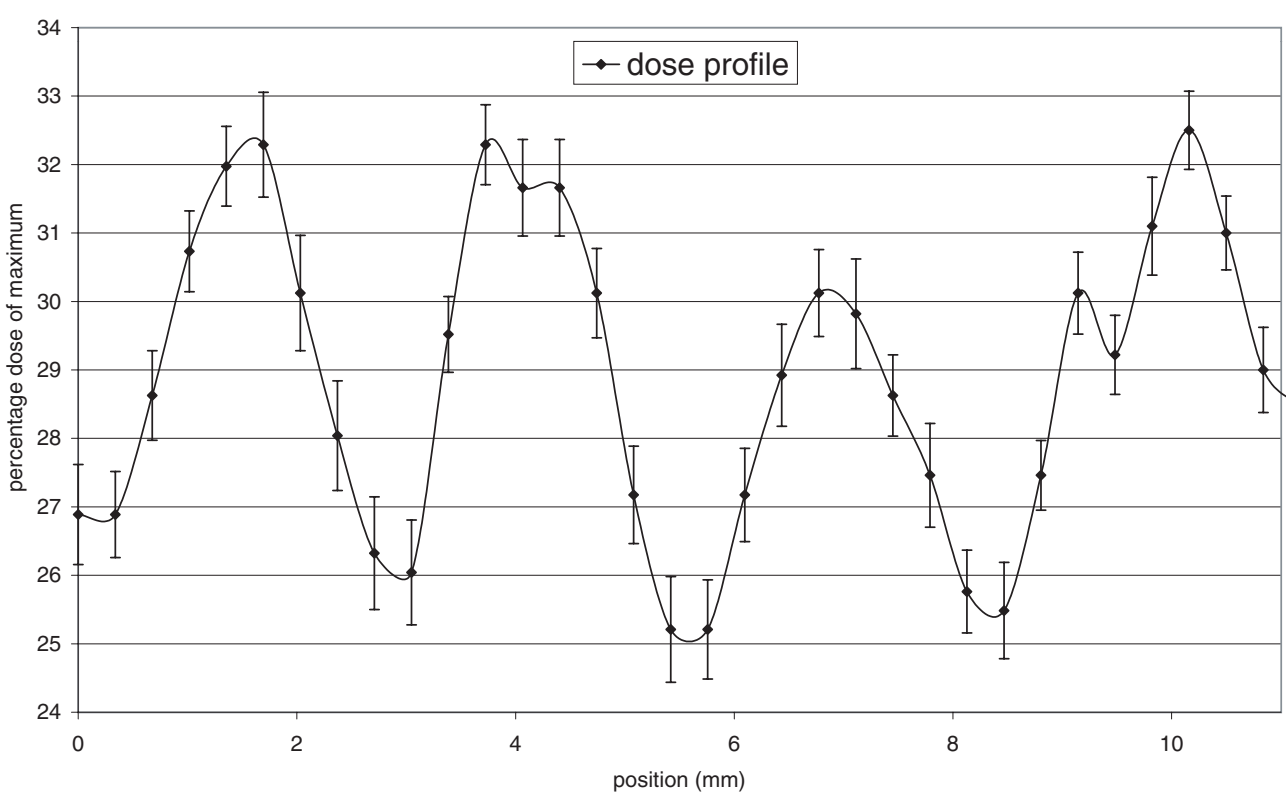

Figure 3. Percentage dose profile measured using Gafchromic film placed directly under the Mepitel dressing.

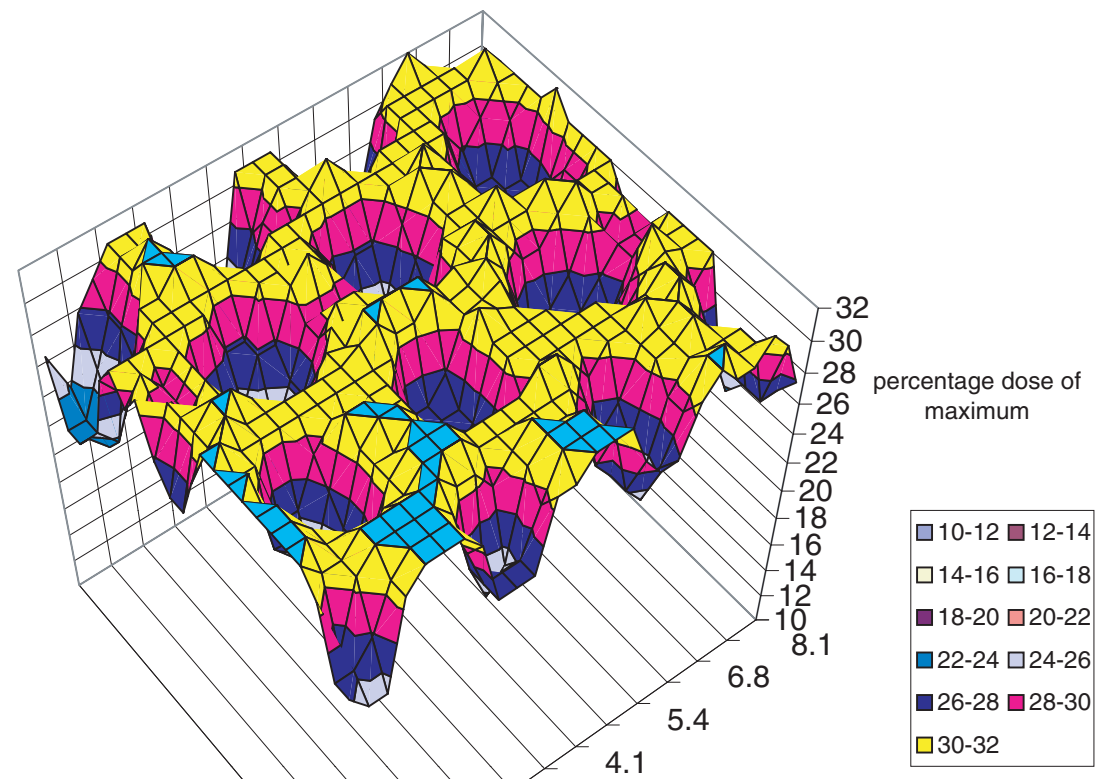

2.7

\section{4}

0.0

Figure 4. A two-dimensional dose map of percentage skin dose measured with Gafchromic film under the Mepitel dressing.

(This figure is in colour only in the electronic version) 
of the maximum and the average increase in dose at $1 \mathrm{~mm}$ depth is $5-6 \%$ of the maximum. There is however a variation in skin dose associated with the mesh pattern of the dressing, with matter directly below the solid part of the dressing receiving a higher dose than do the areas for which the holes are present.

\section{Conclusion}

The measurement of variations in skin dose delivered when using a silicon-based burn dressing has been carried out using TLDs, parallel plate ionization chambers and Gafchromic film. TLDs of various thicknesses and ionization chambers provide point-dose assessment and Gafchromic film can provide a detailed 2D dose map with a high spatial resolution. Increases in skin dose produced by the silicon dressing range from $14 \%$ of the maximum for a $5 \mathrm{~cm} \times$ $5 \mathrm{~cm}$ field at the surface to $4 \%$ of the maximum for a $40 \mathrm{~cm} \times 40 \mathrm{~cm}$ field at $1 \mathrm{~mm}$ depth with a definite variation in delivered dose due to the mesh pattern of the silicon dressing. For assessment of patient skin dose when using a silicon-based burn dressing, the average skin dose over a selected area as well as a 2D map produced by the Gafchromic film would provide a detailed analysis. The effective thickness of the dressing was calculated to be $0.5 \mathrm{~mm}$ water equivalence.

\section{Acknowledgment}

This work has been fully supported by a grant from the Research Grants Council of HKSAR, China (project no CityU 1137/00P).

\section{References}

Butson M, Mathur J, Perez M and Metcalfe P 19966 MV x-ray dose in the build up region: empirical model and the incident angle effect Australas. Phys. Eng. Sci. Med. 19 74-82

Butson M, Yu P and Metcalfe P 1998 Effects of ambient and readout light sources on Gafchromic film Phys. Med. Biol. 43 2407-12

Carolan M G, Butson M J, Herrmann K, Mathur J N and Metcalfe P E 1997 Conversion of an infrared densitometer for radiochromic film analysis Australas. Phys. Eng. Sci. Med. 20 183-5

Constantinou C, Attix F H and Paliwal B R 1982 A solid water phantom material for radiotherapy X-ray and gamma ray beam ray calculations $\mathrm{Med}$. Phys. 9 436-41

Hounsell A R and Wilkinson J M 1999 Electron contamination and build-up doses in conformal radiotherapy fields Phys. Med. Biol. 44 43-55

Kron T, Elliot A, Wong T, Showell G, Clubb B and Metcalfe P 1993 X-ray surface dose measurements using TLD extrapolation Med. Phys. 20 703-11

Meigooni A, Sanders M, Ibbott G and Szeglin S 1996 Dosimetric characteristics of an improved radiochromic film Med. Phys. 23 1883-8

Niroomand-Rad A, Blackwell C, Coursey B, Gall K, Galvin J, McLaughlin W, Meigooni A, Nath R, Rodgers J and Soares C 1998 Radiochromic film dosimetry: recommendation of AAPM radiation therapy task group $55 \mathrm{Med}$. Phys. 25 2093-115

Rawlinson J A, Arlen D and Newcombe D 1992 Design of parallel plate ion chambers for build up measurements in megavoltage photon beams Med. Phys. 19 641-8

Thilmann C, Adamietz I A, Ramm U, Mose S, Saran F and Bottcher H D 1996a Radiation load on the skin using a silicone-coated polyamide wound dressing during photon and electron radiotherapy Strahlenther Onkol 172 $270-4$

Thilmann C, Adamietz I A, Mose S, Saran F, Ramm U and Bottcher H D 1996b Increase of surface dose using wound dressings during percutaneous radiotherapy with photons and electrons Radiother. Oncol. 40 181-4

Vloemens A F and Kreis R W 1994 Fixation of skin grafts with a new silicone rubber dressing (Mepitel) Scand. J. Plast. Reconstr. Surg. Hand. Surg. $2875-6$

Williams C 1995 Mepitel Br. J. Nurs. 4 51-2, 54-5

Zhu T C and Palta J R 1998 Electron contamination in 8 and 18 MV photon beams Med. Phys. 25 12-9 